\title{
Representación de los Equilibrios Líquidos Multifásicos de Sistemas Cuaternarios con los Modelos NRTL y UNIQUAC
}

Fernando García-Sánchez,, (1)* Daimler N. Justo-García, ${ }^{(2), ~ J a c i n t o ~ A ́ g u i l a-H e r n a ́ n d e z ~}{ }^{(1)}$ y Ascención Romero-Martínez ${ }^{(1)}$

(1) Programa de Investigación en Ingeniería Molecular, Instituto Mexicano del Petróleo. Eje Central Lázaro Cárdenas 152, 07730 México, D.F.-México (e-mail: fgarcias@imp.mx; jaherna@imp.mx, aromero@imp.mx)

(2) Departamento de Ingeniería Química Petrolera, ESIQIE, Instituto Politécnico Nacional, Unidad Profesional Adolfo López Mateos, Zacatenco, 07738 México, D.F.-México (e-mail: djusto@ipn.mx)

${ }^{*}$ Autor a quien debe ser dirigida la correspondencia

Recibido Mar. 10, 2011; Aceptado Abr. 28, 2011; Versión Final recibida Jul. 08, 2011

\section{Resumen}

Se presenta el modelado de los equilibrios líquidos multifásicos de sistemas cuaternarios utilizando los modelos de coeficiente de actividad NRTL y UNIQUAC. EI procedimiento numérico utilizado para calcular los equilibrios líquidos multifásicos de estos sistemas, está basado en la minimización de la energía de Gibbs del sistema con pruebas de estabilidad termodinámica para encontrar el estado más estable del sistema. Los algoritmos que se presentan para la estimación de los parámetros de interacción binaria de los modelos NRTL y UNIQUAC fueron aplicados en la correlación de datos experimentales para dos sistemas cuaternarios con dos y tres fases líquidas en equilibrio. La concordancia entre los datos experimentales y los calculados con ambos modelos termodinámicos fue satisfactoria para los dos sistemas estudiados.

\section{Modeling of Multiphase Liquid Equilibria for Quaternary Systems with the NRTL and UNIQUAC Models}

\begin{abstract}
The modeling of multiphase liquid equilibria for quaternary systems using the activity coefficient models NRTL and UNIQUAC is presented. The numerical procedure used to calculate the multiphase liquid equilibria of these systems is based on the minimization of the system Gibbs energy in conjunction with thermodynamic stability tests to find the most stable state of the system. The algorithms presented to estimate the binary interaction parameters of the NRTL and UNIQUAC models were applied to the correlation of the experimental data for two quaternary systems with two and three liquid phases in equilibrium. The agreement between experimental equilibrium data and those calculated with both models was satisfactory for the two systems studied.
\end{abstract}

Keywords: liquid-liquid equilibrium, solution models, Gibbs energy, thermodynamic stability, numerical algorithm 


\section{INTRODUCCION}

Los equilibrios líquidos multifásicos de sistemas multicomponentes son de interés en las industrias química y del petróleo. Sin embargo, estos sólo han sido estudiados con diferentes grados de éxito, por lo que muchos aspectos de ellos aún requieren de mayor investigación. En la industria del petróleo, el diseño de los procesos de recuperación mejorada por químicos y la interpretación de su rendimiento, requiere de un entendimiento comprensivo del comportamiento de fases de los sistemas químicos involucrados. En general, los sistemas que son efectivos en la recuperación del petróleo se caracterizan por la presencia de tres fases líquidas en equilibrio, las cuales aparecen y desaparecen a medida que se varía la concentración de uno o más componentes sobre ciertos intervalos que se encuentran durante el desplazamiento a través de los medios porosos. En la industria química, la tecnología de la extracción por solventes depende en gran medida de la miscibilidad limitada y la distribución de un soluto entre dos fases líquidas. Por ejemplo, en destilación azeotrópica, la separación del componente de arrastre de los productos obtenidos después del condensador se facilita debido a la formación de dos fases líquidas (Cairns y Furzer, 1990). En el diseño del equipo para tales procesos y la simulación de los procesos químicos a escala industrial, es necesario conocer, ya sea de datos experimentales o a través de cálculos, la composición de los componentes de cada fase en equilibrio.

Para calcular los equilibrios líquido-líquido multifásicos, es esencial conocer los potenciales químicos de los componentes en cada fase, así como las estabilidades relativas de las diferentes fases en equilibrio, las cuales están relacionadas a la termodinámica del sistema a través de las funciones de energía de Gibbs de exceso. El problema multifásico es el cálculo más importante en equilibrio de fases, el cual consiste en encontrar el número y tipo de fases correcto y sus correspondientes composiciones al equilibrio, tal que la energía de Gibbs del sistema sea un mínimo. En este aspecto, un gran número de métodos han sido aplicados para resolver el problema del equilibrio de fases y todos estos están basados en resolver una secuencia de subproblemas. Aunque cada método tiene sus propias especificaciones, desde el punto de vista de formulación y solución del problema, los métodos pueden ser agrupados en dos diferentes clases: método de solución de ecuaciones y método de minimización de la energía de Gibbs. En la primera clase de métodos, se resuelve un sistema de ecuaciones no lineales que surge de los balances de masa y de las relaciones de equilibrio de fases, los cuales satisfacen las condiciones necesarias de igualdad de los potenciales químicos de todos los componentes en cada fase (condición necesaria pero no suficiente de equilibrio). En este caso, el objetivo es determinar las composiciones de las fases en equilibrio. Algoritmos tales como el de substitución sucesiva, Newton o cuasi-Newton, han sido utilizados con éxito para resolver el problema de los equilibrios multifásicos por diferentes autores (e.g., Michelsen, 1982b; Stateva et al., 2000; García-Sánchez et al., 2001).

La segunda clase de métodos, consiste en minimizar directamente la energía de Gibbs del sistema, para lo cual una gran variedad de métodos de optimización pueden ser utilizados. Sin embargo, debido a que el número y la identidad de las fases presentes al equilibrio no son conocidas a priori, la aplicación de los métodos de optimización a la solución del problema del equilibrio de fases puede causar severas dificultades computacionales. Para superar este problema, diferentes estrategias han sido propuestas en la literatura. La primer estrategia consiste en fijar el número de fases (dos o tres fases, generalmente, porque son los casos más comunes) y utilizar, por ejemplo, programación no lineal para minimizar la energía de Gibbs para estas fases (Lantagne et al., 1988). En la segunda estrategia, se adiciona secuencialmente una fase extra en los cálculos y se prueba la estabilidad de la configuración de fases resultante (Castier et al., 1989). Sin embargo, esta estrategia puede converger ya sea a extremos locales o a mínimos restringidos si se asumen demasiadas fases (Wakeham y Stateva, 2004). Además, debido a que cualquier solución de equilibrio a la energía de Gibbs debe satisfacer la igualdad de los potenciales químicos, un problema común en todos los métodos de optimización aplicados a la minimización directa de la energía de Gibbs es que para sistemas no ideales pueden existir múltiples soluciones, correspondientes a extremos locales de la función de energía de Gibbs, de las cuales sólo una está en el mínimo global. Por consiguiente, se requiere una condición de suficiencia, la cual es suministrada por el criterio del plano tangente (Baker et al., 1982; Michelsen et al., 1982a). 
En años recientes, diferentes autores han utilizado métodos de optimización global para resolver el problema de los equilibrios entre fases, ya que encontrar el mínimo global es importante porque éste corresponde a la solución correcta del problema (Rangaiah, 2001). Básicamente, el cálculo del equilibrio de fases involucra dos problemas: (1) análisis de estabilidad de fases para determinar si un sistema de prueba, a temperatura y presión específicas, es estable o no, y (2) cálculo de separación de fases para establecer el número e identidad de las fases que coexisten en equilibrio. No obstante, es importante remarcar que el cálculo de los equilibrios de fases se efectúa, generalmente, miles de veces en un simulador de procesos y hasta millones de veces en simulaciones numéricas de procesos de recuperación mejorada del petróleo, por lo que se debe tener precaución en el uso de métodos de optimización global, ya que, a pesar de localizar el mínimo global de la energía de Gibbs, el número de llamadas a la función que se está minimizando a veces resulta prohibitivo. Una excelente revisión sobre la aplicación de los métodos de convergencia local y de optimización global a la solución numérica del problema del equilibrio de fases isotérmico-isobárico, puede encontrarse en el trabajo presentado por Wakeham y Stateva (2004). En la última década, sólo algunos estudios han sido enfocados al cálculo de los equilibrios líquidos multifásicos con modelos de energía de Gibbs de exceso, entre los cuales se encuentran los reportados por Stateva et al. (2000), García-Sánchez et al. (2001), Solokhin et al. (2002), Guo et al. (2004), Denes et al. (2006), Marcilla et al. (2009) y Kangas et al. (2011). Estos autores utilizaron diferentes estrategias numéricas para encontrar la solución del problema del flash isotérmico-isobárico de sistemas ternarios y/o cuaternarios que presentan múltiples fases líquidas.

El propósito del presente trabajo es aplicar los modelos NRTL (Renon y Prausnitz, 1968) y UNIQUAC (Abrams y Prausnitz, 1975) para representar datos de equilibrio líquido-líquido de sistemas cuaternarios con dos y tres fases líquidas en equilibrio, a temperatura y presión específicas, utilizando un método de convergencia local para el cálculo de los equilibrios líquidos multifásicos que incluye pruebas de estabilidad termodinámica y minimización de la energía de Gibbs del sistema.

\section{PROCEDIMIENTO NUMÉRICO}

En este trabajo, se utilizó un eficiente procedimiento computacional para resolver el problema del flash isotérmico multifásico de sistemas multicomponentes con dos modelos de energía de Gibbs de exceso: NRTL y UNIQUAC. En este procedimiento, se asume que el sistema es inicialmente monofásico y una prueba de estabilidad permite verificar si el sistema es estable o no. En caso de que el sistema sea inestable, esta técnica suministra una estimación de la composición de una fase adicional; entonces, se incrementa el número de fases por uno y el equilibrio se alcanza minimizando la energía de Gibbs. Esta aproximación, concebida como un procedimiento sucesivo (Michelsen, 1982b), continúa hasta que se encuentra una solución estable.

En esta técnica, se consideró el análisis de estabilidad de un sistema homogéneo de composición $\mathbf{z}$, basado en la minimización de la distancia que separa la energía de Gibbs del plano tangente en $\mathbf{z}$ (Baker et al., 1982). En términos de coeficientes de actividad, $\gamma_{i}$, este criterio para estabilidad puede escribirse de la forma (Michelsen, 1982a)

$$
\mathrm{F}(\mathbf{x})=\sum_{\mathrm{i}=1}^{\mathrm{N}} \mathrm{x}_{\mathrm{i}}\left[\ln \mathrm{x}_{\mathrm{i}}+\ln \gamma_{\mathrm{i}}(\mathbf{x})-\mathrm{h}_{\mathrm{i}}\right] \geq 0 \quad \forall \mathrm{x}
$$

donde

$$
\mathrm{h}_{\mathrm{i}}=\ln \mathrm{z}_{\mathrm{i}}+\ln \gamma_{\mathrm{i}}(\mathbf{z}) \quad \mathrm{i}=1, \ldots, \mathrm{N}
$$

La Ec. (1) requiere que el plano tangente no caiga, en ningún punto, por arriba de la superficie de la energía de Gibbs y esto se alcanza cuando la función $F(\mathbf{x})$ es positiva en todos sus mínimos. En consecuencia, se debe considerar un mínimo de $F(\mathbf{x})$ en el interior de la región admisible $\sum_{i=1}^{N} x_{i}=1, \forall x \geq 0$. Debido a que no es posible físicamente probar la condición (1) para todas las composiciones de prueba, entonces es suficiente probar la estabilidad en todos los puntos 
estacionarios de $F(\mathbf{x})$, ya que esta función es positiva en todos los puntos estacionarios. No obstante, es importante mencionar que el cambio en la energía será negativo si la función $F(\mathbf{x})$ es negativa, lo que indica que la mezcla original es inestable y susceptible de separarse en dos o más fases.

Un criterio de estabilidad equivalente al de la Ec. (1), pero basado en las variables $\xi_{\mathrm{i}}$ (número de moles del componente $i$ con correspondientes fracciones molares $\left.x_{i}=\xi_{i} / \sum_{j=1}^{N} \xi_{j}\right)$, también puede formularse de la forma (Michelsen, 1982a)

$\mathrm{F}^{*}(\boldsymbol{\xi})=1+\sum_{\mathrm{i}=1}^{\mathrm{N}} \xi_{\mathrm{i}}\left[\ln \xi_{\mathrm{i}}+\ln \gamma_{\mathrm{i}}(\boldsymbol{\xi})-\mathrm{h}_{\mathrm{i}}-1\right] \geq 0 \quad \forall \xi>0$

El método quasi-Newton BFGS (Fletcher, 1980) fue aplicado a la Ec. (3) para determinar la estabilidad de un sistema de composición $\mathbf{z}$, a temperatura y presión especificas. Considerando que el objetivo de minimizar la función $\mathrm{F}^{*}(\boldsymbol{\xi})$ es saber si el sistema es estable o inestable; es decir., encontrar los mínimos negativos de esta función utilizando diferentes inicializaciones de prueba (tantas inicializaciones como componentes en la mezcla). Al final de la minimización de $\mathrm{F}^{*}(\boldsymbol{\xi})$ para las diferentes inicializaciones, se tendrán dos mínimos negativos de la función si el sistema es intrínsecamente inestable y un mínimo negativo si el sistema es metaestable. Cuando se tienen dos mínimos negativos de $\mathrm{F}^{*}(\boldsymbol{\xi})$, entonces los coeficientes de equilibrio se calculan a partir de las composiciones de prueba para cada una de las inicializaciones donde $F^{*}(\boldsymbol{\xi})$ es negativa, mientras que cuando se tiene un sólo mínimo negativo, los coeficientes de equilibrio se calculan a partir de la composición de prueba donde $\mathrm{F}^{*}(\boldsymbol{\xi})$ es negativa y de la composición global de la mezcla. En este caso, los valores de los coeficientes de equilibrio obtenidos a partir de la prueba de estabilidad representan una inicialización robusta al problema del cálculo del equilibrio de fases, ya que estos coeficientes de equilibrio son muy cercanos a los de la solución.

Una vez que se detecta la inestabilidad con la solución a $p-1$ fases, entonces se realiza el cálculo del equilibrio a $\mathrm{P}$ fases mediante la minimización de la energía de Gibbs

$\operatorname{Min}_{n_{i}^{(\varphi)}} G=\sum_{\varphi=1}^{p} \sum_{i=1}^{N} n_{i}^{(\varphi)} \mu_{i}^{(\varphi)}$

sujeto a las restricciones del balance de materia

$\sum_{\varphi=1}^{p} n_{i}^{(\varphi)}=z_{i} \quad i=1, \ldots, N$

y a las restricciones de desigualdad

$n_{i}^{(\varphi)} \geq 0 \quad i=1, \ldots, N ; \varphi=1, \ldots, p$

donde $z_{i}$ es la fracción molar del componente $i$ en el sistema y $n_{i}^{(\varphi)}$ es el número de moles del componente $\mathrm{i}$ en la fase $\varphi$ por mol de alimentación. Expresando los potenciales químicos $\mu_{i}^{(\varphi)}($ $\mathrm{i}=1, \ldots, \mathrm{N} ; \varphi=1, \ldots, \mathrm{p})$ en términos de los coeficientes de actividad y considerando la composición $n_{i}^{(p)}$ como dependiente de las variables $n_{i}^{(\varphi)}(i=1, \ldots, N ; \varphi=1, \ldots, p-1)$, entonces el problema se reduce a la siguiente minimización restringida

$\operatorname{Min}_{n_{i}^{(\varphi)}} \Delta g=\sum_{\varphi=1}^{p} \sum_{i=1}^{N} n_{i}^{(\varphi)} \ln \left(x_{i}^{(\varphi)} \gamma_{i}^{(\varphi)}\right)$

con las restricciones de desigualdad dadas por la Ec. (6) y la restricción

$\sum_{\varphi=1}^{p-1} n_{i}^{(\varphi)} \leq z_{i} \quad i=1, \ldots, N$ 
donde $n_{i}^{(p)}, x_{i}^{(p)}$ y $\gamma_{i}^{(p)}\left(T, P, x^{(p)}\right)$ se consideran funciones de $n_{i}^{(\varphi)}(i=1, \ldots, N ; \varphi=1, \ldots, p-1)$.

La Ec. (7) puede resolverse de manera eficiente utilizando un algoritmo de minimización sin restricciones manteniendo las variables $n_{i}^{(\varphi)}$ dentro del dominio convexo de restricciones dado por las Ecs. (6) y (8) durante la búsqueda de la solución. En este caso, se utilizó una aproximación híbrida para minimizar la Ec. (7), iniciando con el método del máximo descenso junto con una inicialización robusta suministrada de la prueba de estabilidad para asegurar un cierto progreso a partir de las inicializaciones y terminando con el método quasi-Newton, el cual asegura la propiedad del descenso estricto en la superficie de la energía de Gibbs.

Para utilizar un modelo de la energía de Gibbs de exceso para el cálculo de los equilibrios líquidolíquido, es esencial obtener los parámetros ajustables del modelo a partir de la regresión de los datos experimentales, los cuales, a su vez, pueden ser utilizados para la interpolación de los datos o extrapolaciones en regiones más allá donde las mediciones fueron realizadas. Por lo tanto, con un conjunto dado de parámetros del modelo, es posible calcular el número de fases en equilibrio y sus composiciones a partir de la composición global del sistema. Sin embargo, si la minimización de $\Delta g$ se inicia a partir de cualquier conjunto de parámetros, el cálculo de los equilibrios entre fases pudiera conducir a un sistema homogéneo o a un sistema de fases múltiples, el cual sería diferente al número de fases experimentales del sistema de estudio. Por consiguiente, es necesario inicializar estos parámetros con valores razonables. Con esta finalidad, se presentan a continuación los procedimientos utilizados para estimar los parámetros del modelo a partir de datos de equilibrio líquido-líquido.

(1). Minimización de la suma de las diferencias entre los logaritmos de las actividades de cada componente en cada fase,

$F_{a}=\sum_{j=1}^{N e q} \sum_{i=1}^{N} w_{i j}\left[\left(\ln a_{i j}^{\prime}-\ln a_{i j}^{\prime \prime}\right)^{2}+\left(\ln a_{i j}^{\prime}-\ln a_{i j}^{I I I}\right)^{2}\right]+Q \sum_{m=1}^{N p a r} p_{m}^{2}$

donde $\mathrm{w}_{\mathrm{ij}}$ es el factor de peso asociado al componente $\mathrm{i}$ de la línea de unión (o triángulo de unión) $\mathrm{j}$ y $\mathrm{a}_{\mathrm{ij}}^{1}=\mathrm{x}_{\mathrm{ij}}^{\mathrm{l}} \gamma_{\mathrm{ij}}^{\mathrm{l}}$ es la actividad del componente $\mathrm{i}$ de la línea de unión (o triángulo de unión) j en la fase $\mathrm{I}$; siendo $x_{i j}^{\prime}$ la fracción molar experimental correspondiente y $\gamma_{i j}^{\prime}$ el coeficiente de actividad, el cual es calculado a partir de un modelo de energía de Gibbs de exceso que depende de $x_{\mathrm{ij}}^{\mathrm{i}}$ y de los parámetros del modelo $\mathrm{p}_{\mathrm{m}}(\mathrm{m}=1, \ldots, \mathrm{Npar})$.

(2). Minimización de la suma de las diferencias entre las fracciones molares experimentales y las calculadas,

$F_{x}=\sum_{k=1}^{N e q} \sum_{j=1}^{N p h} \sum_{i=1}^{N} w_{i j k}\left(x_{i j k}^{\text {expl }}-x_{i j k}^{\text {calc }}\right)^{2}+Q \sum_{m=1}^{N p a r} p_{m}^{2}$

donde $\mathrm{w}_{\mathrm{ijk}}, \mathrm{x}_{\mathrm{ijk}}^{\mathrm{expl}}, \mathrm{y} \mathrm{x}_{\mathrm{ijk}}^{\text {calc }}$ representan, respectivamente, el factor de peso, la fracción molar experimental y la fracción molar calculada del componente $i$ en la fase $j$ correspondiente a la línea de unión (o triángulo de unión) k .

El segundo término en el lado derecho de las Ecs. (9) y (10), es agregado a las funciones objetivo $F_{a}$ y $F_{x}$ para asegurar que se tengan parámetros relativamente pequeños sin incrementar el mínimo de estas funciones y así evitar el riesgo de soluciones múltiples. Este término también tiene la ventaja de que el mínimo de $\mathrm{F}_{\mathrm{a}}$ y $\mathrm{F}_{\mathrm{x}}$ se hace más acentuado, promoviendo así la convergencia, y es activado sólo si uno o más parámetros del modelo son mayores a un valor específico. En este trabajo, el valor de la constante $Q$ fue elegido de tal manera que la cantidad $\mathrm{Q}(500)^{2}$ fuera el uno por ciento del valor de la función objetivo $\left(\mathrm{F}_{\mathrm{a}} \circ \mathrm{F}_{\mathrm{x}}\right)$. Esta aproximación fue utilizada en todos los casos para estimar los parámetros de interacción del modelo de energía de 
Gibbs de exceso. Además, se reemplazaron las incertidumbres experimentales (las cuales, en general, no están disponibles) por factores de peso que pueden ser utilizados para forzar el modelo termodinámico representar ciertas composiciones con más exactitud. Estos factores fueron puestos igual a la unidad durante la correlación de los datos de equilibrio líquido estudiados en este trabajo.

Aunque la función objetivo $F_{a}$ ha sido utilizada frecuentemente en la literatura, ésta presenta la desventaja de que sólo contiene las diferencias entre las actividades calculadas, por lo que la minimización de esta función no necesariamente conduce a pequeñas diferencias entre las fracciones molares experimentales y calculadas, lo que es deseable en la práctica. No obstante, para obtener un valor inicial de los parámetros del modelo, es con frecuencia útil utilizar la función $F_{a}$ ya que ésta puede ser evaluada explícitamente. Los parámetros obtenidos de esta manera procuran un estimado inicial lo suficientemente aceptable para permitir una convergencia menos onerosa en las iteraciones subsecuentes basadas en la función $F_{x}$.

Por otra parte, la minimización de la función $F_{x}$ es más complicada y consume más tiempo en su evaluación, ya que ésta involucra el cálculo de las fracciones molares $x_{\mathrm{ijk}}^{\text {calc }}$ para un conjunto dado de parámetros de los cuales surgirá un nuevo conjunto de parámetros. El cálculo continua hasta que el valor de $F_{x}$ sea menor al de una tolerancia prescrita o cuando se ha alcanzado el número máximo de iteraciones.

En la Ec. (10) las fases experimentales y calculadas correspondientes a cada punto de composición global, son acopladas por orden de proximidad decreciente. Sin embargo, es posible que durante la minimización de la función $F_{x}$ exista un mal acoplamiento en el número de fases experimentales y calculadas; e.g., cuando hay dos fases experimentales y tres fases calculadas. En este caso, la fase calculada más alejada de las fases experimentales no se considera en la evaluación de la función objetivo. De hecho, se espera que esta última fase sólo aparezca en una cantidad muy pequeña a medida que las otras fases calculadas estén cercanas a las fases experimentales, ya que los sistemas experimentales y calculados corresponden a la misma composición global. En este aspecto, esta aproximación siempre acopló correctamente el número de fases experimentales y calculadas para los sistemas estudiados.

En este trabajo, los parámetros de interacción de los modelos NRTL y UNIQUAC fueron obtenidos a través de la minimización de la funciones $F_{a}$ y $F_{x}$ con el método de optimización simplex (Nelder y Mead, 1965).

\section{RESULTADOS Y DISCUSIÓN}

Los métodos descritos anteriormente para el cálculo de los equilibrios líquidos multifásicos y la estimación de los parámetros de un modelo dado a partir de datos experimentales mostrando dos y/o tres fases líquidas en equilibrio, fueron utilizados en la representación del comportamiento de fases de sistemas cuaternarios con los modelos NRTL y UNIQUAC.

Para ilustrar la capacidad correlativa de estos modelos termodinámicos, se llevó a cabo el modelado de dos sistemas cuaternarios (benceno-heptano-acetonitrilo-agua y benceno-heptanosulfolano-agua) que muestran dos y tres fases líquidas en equilibrio para diferentes composiciones globales. Estos sistemas fueron determinados experimentalmente por Hartwig et al. (1955) a $298.15 \mathrm{~K}$. En el caso del sistema benceno-heptano-acetonitrilo-agua, se determinaron 6 líneas de unión (dos fases líquidas en equilibrio) y 11 triángulos de unión (tres fases líquidas en equilibrio), mientras que para el sistema benceno-heptano-sulfolano-agua, se determinaron 12 líneas de unión y 1 triángulo de unión. Los resultados de la representación de estos sistemas con los modelos NRTL y UNIQUAC se presentan en la Tabla 1.

La comparación entre las fracciones molares experimentales y calculadas se realizó a través del valor de la desviación cuadrática promedio, definida de la forma 


$$
\sigma_{\mathrm{x}}=\sqrt{\frac{\min \left(\mathrm{F}_{\mathrm{x}}\right)}{\left(2 \mathrm{~N}_{\mathrm{bin}}+3 \mathrm{~N}_{\text {tri }}\right) \mathrm{N}-\mathrm{N}_{\mathrm{par}}}}
$$

donde $\mathrm{N}$ es el número de componentes, $\mathrm{N}_{\text {bin }}$ es el número de líneas de unión, $\mathrm{N}_{\text {tri }}$ es el número de triángulos de unión, $\mathrm{N}_{\mathrm{par}}$ es el número de parámetros ajustados del modelo y $\mathrm{F}_{\mathrm{x}}$ es el valor de la función objetivo, obtenida al final de la correlación. La Tabla 1 presenta los valores (en \%) de las desviaciones cuadráticas promedio obtenidas para los sistemas cuaternarios estudiados con los modelos NRTL y UNIQUAC.

Un análisis de las desviaciones cuadráticas promedio reportadas en la Tabla 1, indica que el modelo UNIQUAC representa mejor el sistema benceno-heptano-acetonitrilo-agua, mientras que el desempeño del modelo NRTL fue superior al modelo UNIQUAC en la representación del sistema benceno-heptano-sulfolano-agua; sin embargo, es importante mencionar que ambos modelos son capaces de representar satisfactoriamente el complejo comportamiento de fases que exhiben estos sistemas.

Tabla 1. Parámetros de interacción estimados de los modelos NRTL y UNIQUAC para los sistemas benceno-heptano-acetonitrilo-agua y benceno-heptano-sulfolano-agua a $298.15 \mathrm{~K}$.

\begin{tabular}{|c|c|c|c|c|c|c|c|c|c|c|}
\hline \multirow[b]{2}{*}{ Componente } & \multicolumn{2}{|c|}{$\begin{array}{l}\text { Parámetros } \\
\text { Comp. puro }\end{array}$} & \multirow[b]{2}{*}{$\mathrm{i}, \mathrm{j}$} & \multicolumn{2}{|c|}{$\begin{array}{r}\text { Parámetros } \\
\text { UNIQUAC, K }\end{array}$} & \multirow[b]{2}{*}{$\% \sigma_{x}$} & \multicolumn{3}{|c|}{$\begin{array}{l}\text { Parámetros } \\
\text { NRTL, K }\end{array}$} & \multirow[b]{2}{*}{$\% \sigma_{x}$} \\
\hline & 1 & $q$ & & $a_{i j}$ & $a_{j i}$ & & $\alpha_{i j}$ & $a_{i j}$ & $a_{\mathrm{ji}}$ & \\
\hline Benceno (1) & 3.19 & 2.40 & 1,2 & -99.762 & -42.424 & 1.56 & 0.2 & 1271.7 & -736.17 & 2.80 \\
\hline Heptano (2) & 5.17 & 4.40 & 1,3 & -15.084 & -146.01 & & 0.2 & -364.00 & 313.76 & \\
\hline Acetonitrilo (3) & 1.87 & 1.72 & 1,4 & 1126.3 & 1076.6 & & 0.2 & 1375.8 & 1679.4 & \\
\hline \multirow[t]{3}{*}{ Agua (4) } & 0.92 & 1.40 & 2,3 & 635.19 & 39.090 & & 0.2 & 532.44 & 760.66 & \\
\hline & & & 2,4 & 1572.6 & 565.68 & & 0.2 & 575.96 & 2130.7 & \\
\hline & & & 3,4 & 65.040 & 193.03 & & 0.2 & 34.528 & 666.77 & \\
\hline Benceno (1) & 3.19 & 2.40 & 1,2 & 138.64 & -184.49 & 1.22 & 0.2 & 481.44 & -609.56 & 0.58 \\
\hline Heptano (2) & 5.17 & 4.40 & 1,3 & -5.0461 & 7.9019 & & 0.2 & 374.16 & -329.15 & \\
\hline Sulfolano (3) & 4.04 & 3.20 & 1,4 & 665.75 & 108.51 & & 0.2 & 1312.4 & 2888.5 & \\
\hline \multirow[t]{3}{*}{ Agua (4) } & 0.92 & 1.40 & 2,3 & 836.41 & 45.652 & & 0.2 & 2301.6 & 831.18 & \\
\hline & & & 2,4 & 1047.5 & 865.51 & & 0.2 & 1235.8 & 1701.9 & \\
\hline & & & 3,4 & 372.32 & -188.51 & & 0.2 & 17.770 & 275.73 & \\
\hline
\end{tabular}

Las Figuras 1a-1c presentan los datos de equilibrio líquido-líquido experimentales y los valores calculados de las composiciones en fracción molar para cada uno de los componentes del sistema benceno-heptano-acetonitrilo-agua en las fases inferior (fase acuosa), intermedia y superior (fase orgánica), con el modelo UNIQUAC.

Una observación visual de estas figuras indica que las composiciones calculadas de las fases acuosa (Figura 1a) y orgánica (Figura 1c) están en excelente concordancia con los datos experimentales para todos los componentes del sistema, mientras que algunas fracciones molares para el acetonitrilo y el agua en la fase intermedia (Fig. 1b) muestran una ligera diferencia con respecto a los valores experimentales. 


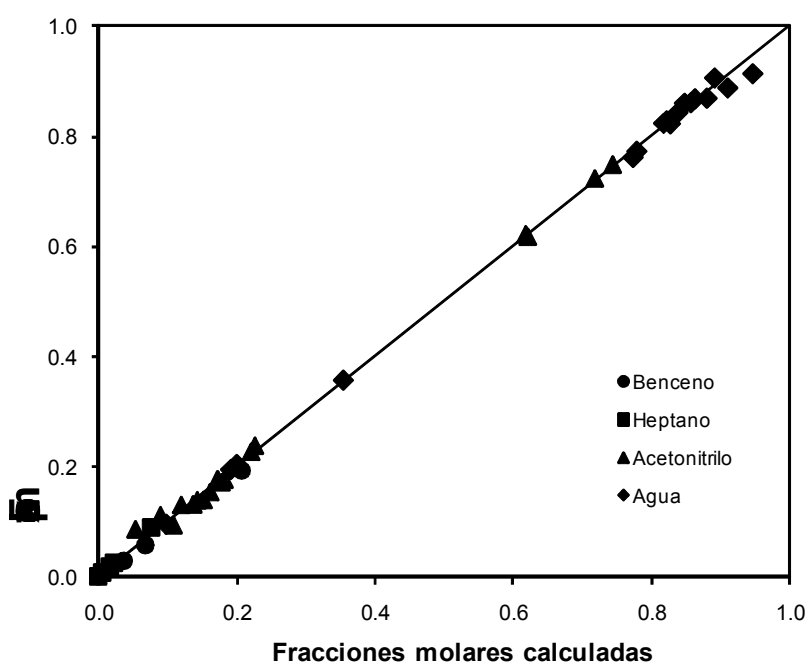

Fig. 1a. Fracciones molares experimentales y calculadas con el modelo UNIQUAC para el sistema benceno-heptano-acetonitrilo-agua a $298.15 \mathrm{~K}$ de la fase acuosa (fase inferior).

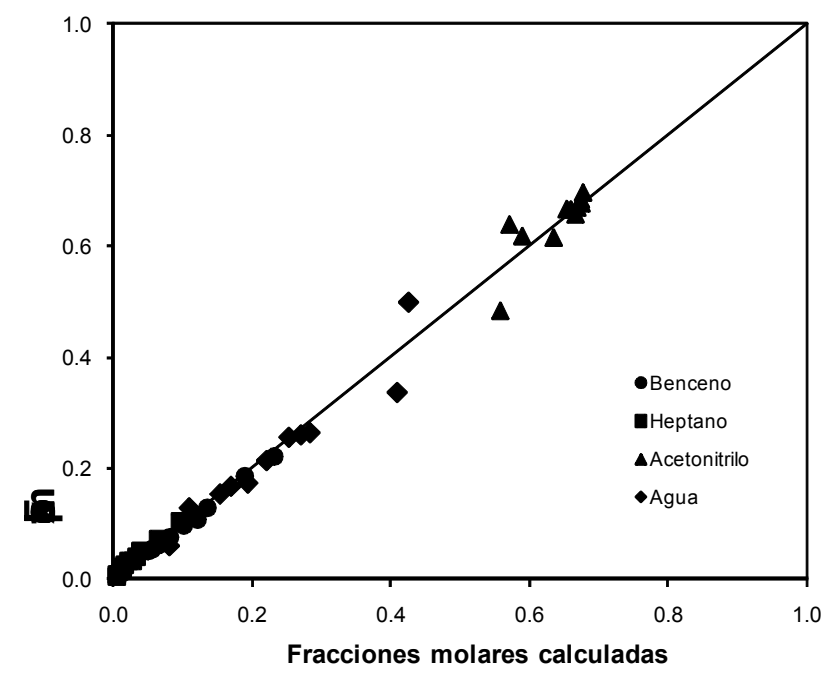

Fig. 1b. Fracciones molares experimentales y calculadas con el modelo UNIQUAC para el sistema benceno-heptano-acetonitrilo-agua a $298.15 \mathrm{~K}$ de la fase intermedia.

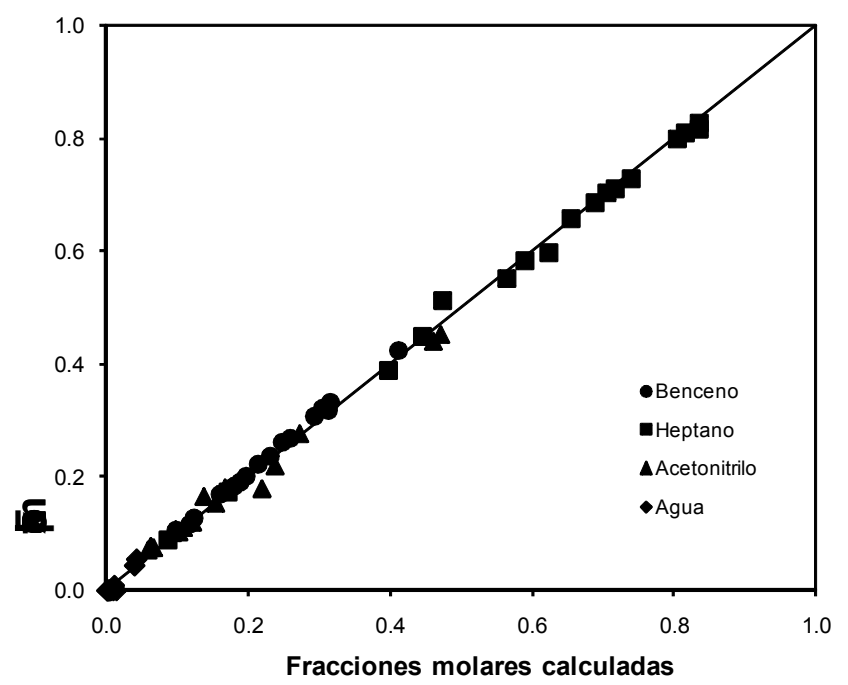

Fig. 1C. Fracciones molares experimentales y calculadas con el modelo UNIQUAC para el sistema benceno-heptano-acetonitrilo-agua a $298.15 \mathrm{~K}$ de la fase orgánica (fase superior). 


\section{CONCLUSIONES}

Se aplicó un eficiente procedimiento numérico para calcular los equilibrios líquidos multifásicos de sistemas multicomponentes a partir de la composición global del sistema, a temperatura y presión especificas. El procedimiento utiliza el criterio del plano tangente para el análisis de estabilidad y una aproximación robusta basada en la minimización de la energía de Gibbs para el cálculo de los equilibrios entre fases.

Este procedimiento, acoplado a un método de correlación de datos, permitió verificar que los modelos NRTL y UNIQUAC representan correctamente el comportamiento de fases de sistemas cuaternarios con dos y tres fases líquidas en equilibrio. En particular, se obtuvo una satisfactoria representación de las composiciones correspondientes a los componentes en las diferentes fases líquidas en equilibrio para los sistemas benceno-heptano-acetonitrilo-agua y benceno-heptanosulfolano-agua, determinados a $298.15 \mathrm{~K}$.

\section{AGRADECIMIENTOS}

Los autores agradecen al Instituto Mexicano del Petróleo por el apoyo prestado para la realización de este trabajo a través del proyecto D.00406.

\section{REFERENCIAS}

Abrams, D. S. y J. M. Prausnitz, Statistical Thermodynamics of Liquid Mixtures: A New Expression for the Excess Gibbs Energy of Partly or Completely Miscible Systems, AIChE J., 21, 116-128 (1975).

Baker, L. E., A. C. Pierce y K. D. Luks, Gibbs Energy Analysis of Phase Equilibria, Soc. Pet. Eng. J., 22, 731-742 (1982).

Cairns, B. P. y I. A. Furzer, Multicomponent Three-Phase Azeotropic Distillation. 2. Phase-Stability and Phase-Splitting Algorithms, Ind. Eng. Chem. Res., 29, 1364-1382 (1990).

Castier, M., P. Rasmusen y A. Fredenslund, Calculation of Simultaneous Chemical and Phase Equilibria in Nonideal Systems, Chem. Eng. Sci., 44, 237-248 (1989).

Denes, F., P. Lang y M. Lang-Lazi, Liquid-Liquid-Liquid Equilibrium Calculations. IChemE Symp. Ser., No. 152, 877-890 (2006).

Fletcher, R., Practical Methods of Optimization. Vol. 1. Unconstrained Optimization, WileyInterscience, Nueva York, USA (1980).

García-Sánchez, F., G. Eliosa-Jiménez, A. Salas-Padrón, O. Hernández-Garduza y D. ÁpamMartínez, Modeling of Microemulsion Phase Diagrams from Excess Gibbs Energy Models, Chem. Eng. J., 84, 257-274 (2001).

Guo, M., S. Wang, J.-U. Repke y G. Wozny, A Simultaneous Method for Two- and Three-Liquid Phase Stability Determination, AIChE J., 50, 2571-2582 (2004).

Hartwig, G. M., G. C. Hood y R. L. Maycock, Quaternary Liquid Systems with Three Liquid Phases, J. Phys. Chem., 58, 52-54 (1955).

Kangas, J., I. Malinen, y J. Tanskanen, Modified Bounded Homotopies in the Solving of Phase Stability Problems for Liquid-Liquid Phase-Splitting Calculations, Ind. Eng. Chem. Res., 50, 70037018 (2011).

Lantagne, G., B. Marcos, y B. Carroll, Computation of Complex Equilibria by Nonlinear Optimization, Comput. Chem. Eng., 12, 589-599 (1988). 
Marcilla, A., M. M. Olaya, M. D. Serrano, R. Velasco y J. A. Reyes-Labarta, Gibbs Energy Based Procedure for the Correlation of Type 3 Ternary Systems Including a Three-Liquid Phase Region, Fluid Phase Equilib., 281, 87-95 (2009).

Michelsen, M. L., The Isothermal Flash Problem. Part I. Stability, Fluid Phase Equilib., 9, 1-19 (1982a).

Michelsen, M. L., The Isothermal Flash Problem. Part II. Phase-Split Calculation, Fluid Phase Equilib., 9, 21-40 (1982b).

Nelder, J. A. y R. A. Mead, Simplex Method for Function Minimization, Comput. J., 7, 308-313 (1965).

Renon, H. y J. M. Prausnitz, Local Composition in Thermodynamic Excess Functions for Liquid Mixtures, AIChE J., 14, 135-144 (1968).

Rangaiah, G. P., Evaluation of Genetic Algorithms and Simulated Annealing for Phase Equilibrium and Stability Problems, Fluid Phase Equilib., 187-188, 83-109 (2001).

Stateva, R. P., G. St. Cholakov, A. A. Galushko y W. A. Wakeham, A Powerful Algorithm for Liquid-Liquid-Liquid Equilibria Predictions and Calculations. Chem. Eng. Sci., 55, 2121-2129 (2000).

Solokhin, M. A., A. V. Solokhin, y U. S. Timofeev, Calculations of Multiphase Liquid-Liquid Equilibrium and Automated Synthesis of the Structures of Phase Diagrams. Theoret. Found. Chem. Eng., 36, 564-569 (2002).

Wakeham, W. A. y R. P. Stateva, Numerical Solution of Isothermal, Isobaric Phase Equilibrium Problem, Rev. Chem. Eng., 20, 1-56 (2004). 